\title{
Sever disease complicated with osteomyelitis, is it role for ultrasonography; case report and review of literature
}

\begin{abstract}
When a physically active growing child presented with heel pain without specific radiographic findings, Sever's disease is usually perused. Sever's disease or calcaneal apophysitis is a subset of osteochondrosis occurring at the posterior calcaneal apophysis at the site of attachment of Achiles tendon. The diagnosis is mainly based on the clinical assessment. Imaging may be needed to exclude alternative diagnosis such as planter fasciitis, Achilles tendon Injuries, calcaneus fractures, osteomyelitis $\&$ tarsal coalition. No sign has been accepted as pathognomonic for sever diagnosis by plain radiography. We present a case of Sever's disease complicated with calcaneal osteomyelitis and discussed the role of ultrasonography.
\end{abstract}

Keywords: osteomyelitis, sever disease, Ultrasonographic role in diagnosis
Volume 8 Issue 4 - 2018

\author{
Manal Elmeniawy,' Mervat Essam,'Ahmed \\ Mohamed Abd elghani,' Rasmia M El- \\ Gohary,' Rana Awad' \\ 'Rheumatology \& Clinical Immunology Unit, Internal Medicine \\ Department, Cairo University, Egypt \\ ${ }^{2}$ Internal Medicine Department, Cairo University, Egypt
}

\begin{abstract}
Correspondence: Mervat Essam, Rheumatology \& Clinical Immunology Unit, Internal Medicine Department, Faculty of Medicine, Cairo University, Egypt, Tel + 20 I 12420507I, Emaillambaa2020@yahoo.com
\end{abstract}

Received: June 30, 2018 | Published: July 26, 2018

\section{Introduction}

When a physically active growing child presented with heel pain without specific radiographic findings, Sever's disease is usually perused. ${ }^{1}$ Sever's disease or calcaneal apophysitis is a subset of osteochondrosis occurring at the posterior calcaneal apophysis at the site of attachment of Achilles tendon. It usually affect the growing skeleton as a result of overuse injuries. ${ }^{2}$ Sever's disease is a self-limiting condition that usually respond well to conservative measures and rarely complicate. We present a case of Sever's disease complicated with calcaneal osteomyelitis and discussed the role of ultrasonography.

\section{Case report}

A well-built 14 years old boy presented with severe constant right heel pain which was increased by physical activity, and relieved

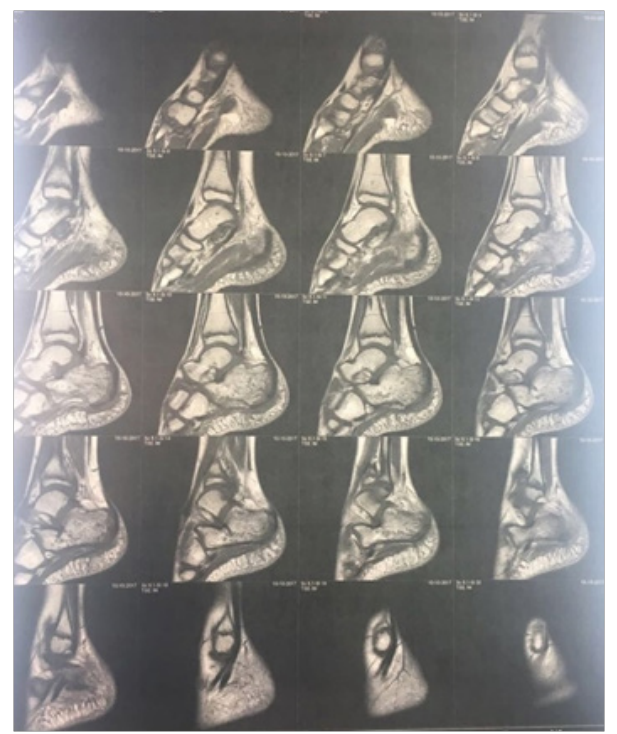

partially by NSAIDs. He was physically active. The patient denied trauma or fever. He reported no history of other musculoskeletal symptoms. At that time physical examination was apparently normal apart from mild swelling and localized tenderness over the posterior right heel. Laboratory tests were significant for elevated ESR (69 $\mathrm{mm})$ and CRP $(12.5 \mathrm{mg} / \mathrm{dl})$. CBC, liver \& kidney function tests were normal. RF was negative. X-ray heel findings showed fragmentation \&increased density of calcaneal apophysis. Although the patient's clinical history, the overuse of sports activity, together with the radiographical findings were in line with calcaneal apophysitis (sever's disease), the unexplained elevation of ESR \& CRP made us to proceed to magnetic resonance imaging (MRI)to exclude osteomyelitis. The MRI demonstrated oedema of the calcaneal apophysis with patchy oedema of the calcaneal bone as well as the overlying soft tissue that consistent with Sever disease (Figure 1). Oral anti inflammatory medication was prescribed in addition the patient was advised to use heel raise shoe orthoses and to restrict sports for 6weeks.

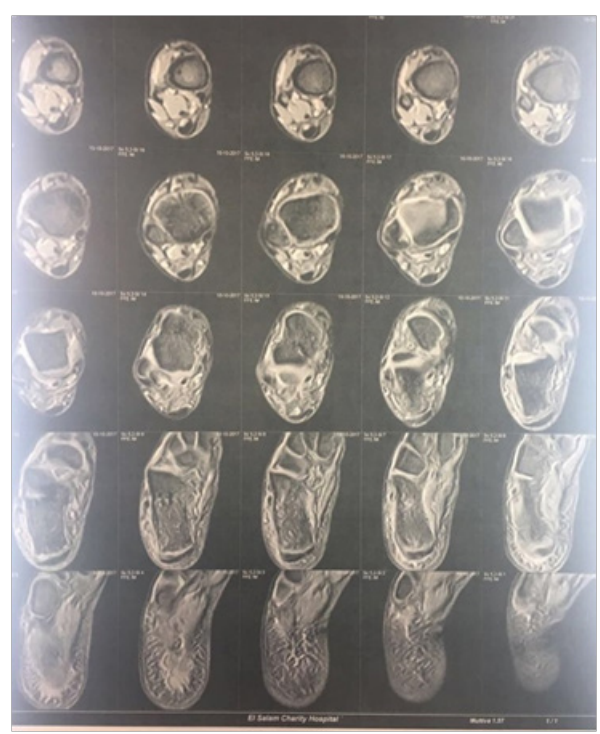



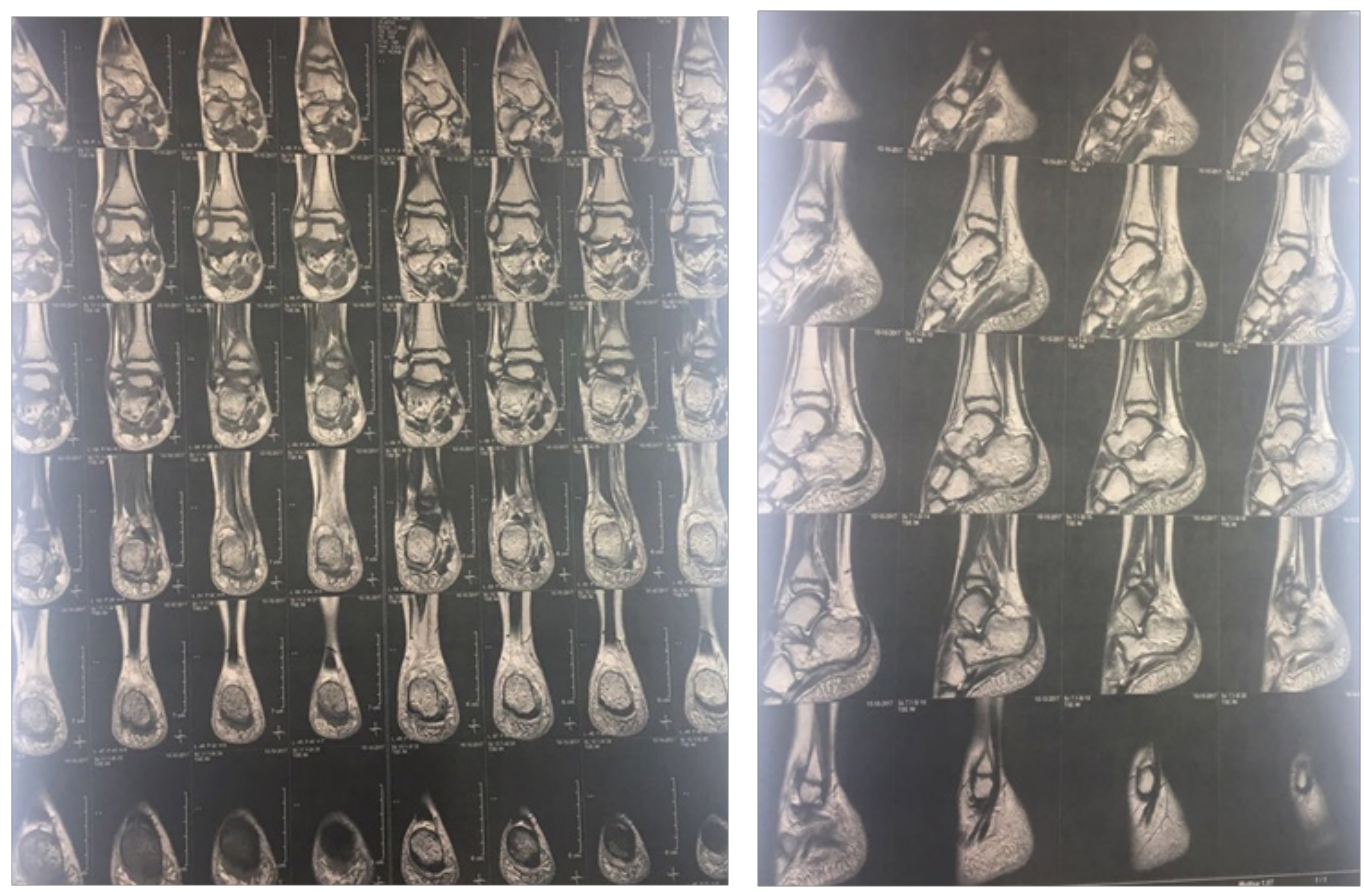

Figure I legend MRI demonstrated oedema of the calcaneal apophysis with patchy oedema of the calcaneal bone as well as the overlying soft tissue.

3 weeks later, the pain became more intense \& agonizing. Her mother reported that her son returned playing foot ball with bare foot. On examination the right posterior heel was swollen, red and tender (Figure 2). This represent a great challenge, being the osteomyelitis provides itself again as a strong diagnosis. Although the MRI is the best available imaging for detecting early osteomyelitis, we used the ultrasonography together with X-ray as preliminary study before considering re-MRI.

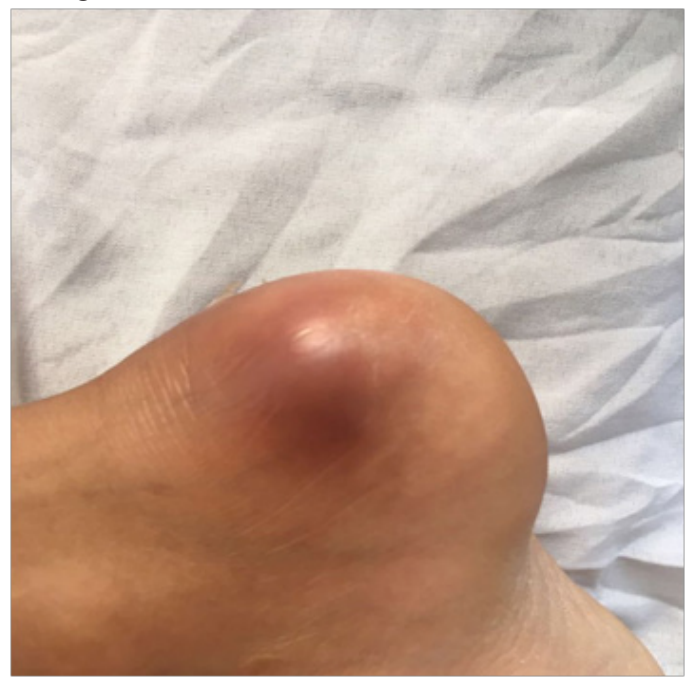

Figure 2 legend Photograph of the patient presenting with right heel pain.

The ultrasonography demonstrated fragmentation of the apophysis of the calcaneus and an avulsed bone fragment at the insertion of the Achilles tendon (Figure 3) together a well-defined echogenic fluid collection with high Doppler signals on both sides of tendo-achilles (Figure 4). Ultrasonographic guided needle aspiration of the fluid collection was performed, the Culture of the aspirated fluid revealed
Staphylococcus aureus. The X-ray showed Fragmentation of calcaneal apophysis \& few osteolytic lesions (Figure 5). Echocardiography was normal. Further evacuation was done surgically and antibiotic was started with marked improvement of the patient condition.

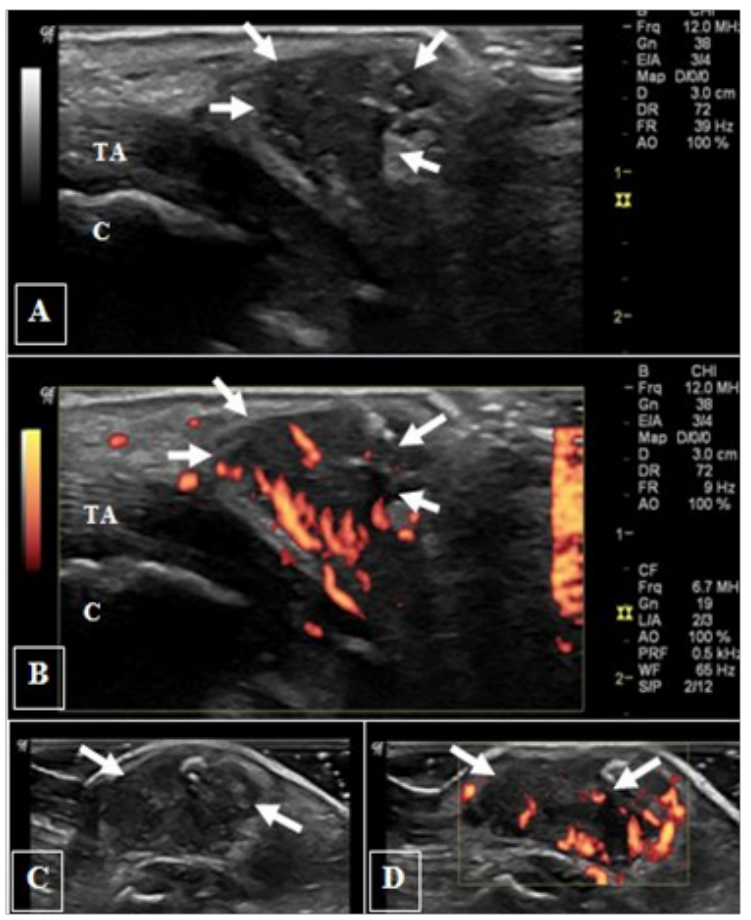

Figure 3 legend (A) Long-axis and (B)short-axis ultrasonography on right heal shows marked cortical irregularity and fragmentation of the posterior calcaneal surface (arrow head) and an avulsed bone fragment (arrow). (C) Long-axis and (D) short-axis ultrasonography on left asymptomatic heal for comparison; note the almost smooth cortical calcaneal surface with subtle irregularity.TA: tendoachieles; C: calcaneal bone. 


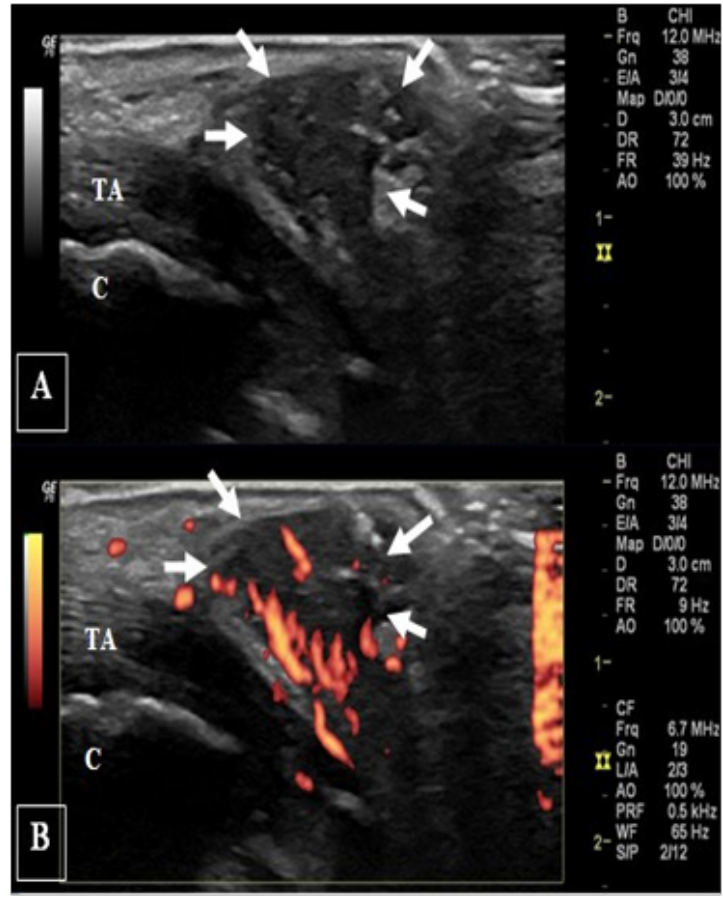

Figure 4 legend (A) Gray-scale and (B) power Doppler long-axis ultrasonography on right heal show well delineated soft tissue collection (arrows) on the side of tendoachiles with increased vascularity. (C) \& (D) represent gray-scale and power Doppler short-axis views respectively on the soft tissue collection. TA: tendoachieles; C: calcaneal bone.

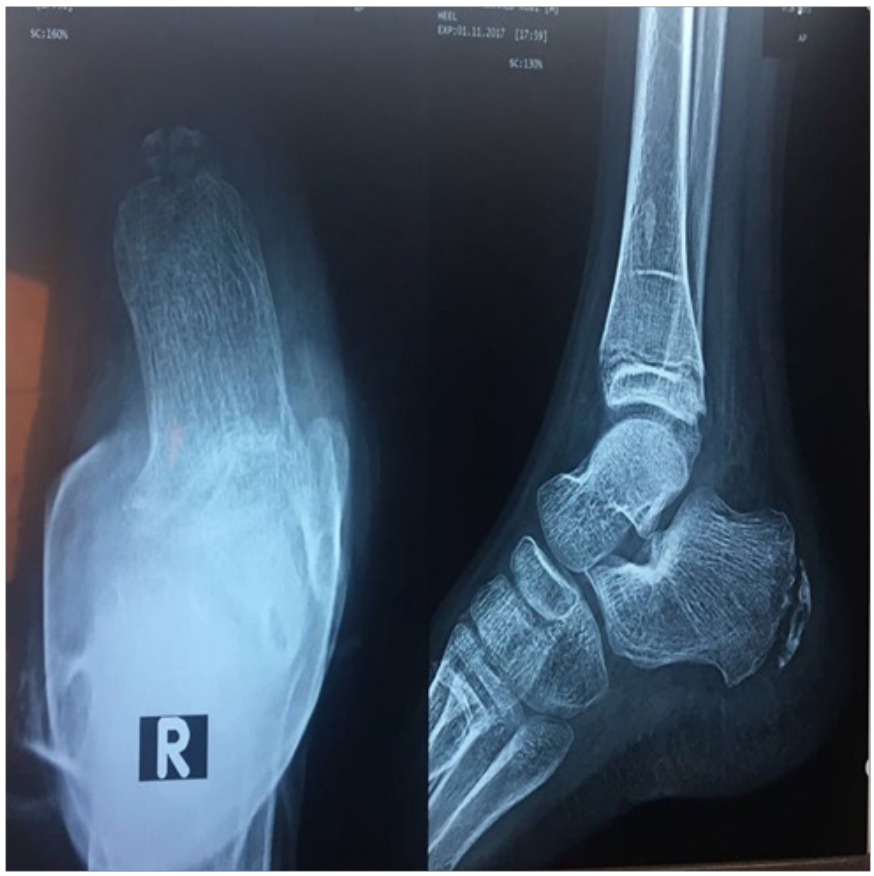

Figure 5 legend $\quad$ x-ray of Rt heel show evidence of osteomyelitis.

\section{Discussion}

Calcaneal apophysitis is a traction overuse syndrome, which commonly occurs in growing children \& adolescents due to repeated microtrauma by the pull of the Achilles tendon on the weak unossified apophysis. $^{3}$
It is similar to tibial tuberosity apophysitis, a condition also known as Osgood-Schlatter disease (OSD) ${ }^{4}$ However unlike the OSD, in which the distal patellar tendon is commonly affected, the involvement of Achilles tendon and retro-calcaneal bursa is not a consistent feature of Sever's disease. ${ }^{2}$

The diagnosis is mainly based on the clinical assessment. The typical symptom includes a localized pain at the postero-plantar aspect of the calcaneus that is exacerbated by motion. On examination there is usually no edema, erythema or warmth. The pain is elicited on medial-lateral squeeze test of the calcaneus in the area of the growth plate. $^{5}$

Imaging may be needed to exclude alternative diagnosis such as planter fasciitis, Achilles tendon Injuries, calcaneus fractures, osteomyelitis \& tarsal coalition. No sign has been accepted as pathognomic for sever diagnosis by plain radiography. Increased density (sclerosis) and fragmentation of the apophysis are demonstrated as two diagnostic radiologic signs. However, Some authors showed that these findings can be found in healthy children. ${ }^{5}$

Inflammatory findings like bone marrow edema of the calcaneal apophysis and enhancement after gadolinium administration can be detected with MRI. ${ }^{6}$ The MRI is mainly used in cases of high suspicion for ruling out osteomyelitis. Over the past decade, musculoskeletal ultrasonography has become incorporated into the rheumatology practice. It has a lower cost, does not use ionizing radiation and offers real-time imaging. The ultrasonography can effectively and safely assess soft tissue lesions that may contribute to the heal pain, including planter fasciitis, Achiles tendon injuries and retro calcaneal bursitis. ${ }^{7,8}$

Hosgören et al., ${ }^{4}$ presumed that ultrasonography may be valuable in the diagnosis of Sever's disease, through its ability to demonstrate calcaneal fragmentation similar to conventional radiograph. ${ }^{4}$ On ultrasonography, the normal surface of a cortical bone appears as a regular hyperechoic line with posterior shadowing and reverberation artifacts. The ultrasonography has higher sensitivity in detection of bone surface abnormalities like erosions, osteophytes and enthesophytes, compared to X-ray. However it could not determine the underlying pathology of cortical irregularities; whether caused by multiple erosions, multiple enthesophytes, osteoarthritic changes or periosteal reaction. Such finding should be interpreted carefully in the context of the patient medical background and his plain radiography. ${ }^{9}$

Sever's disease usually resolves within few weeks to months of initiating conservative treatment, which may include rest, ice application, and heel lift, stretching, and strengthening exercises. Nonsteroidal anti-inflammatory drugs may be beneficial in more severe cases. ${ }^{3}$ Although Sever's disease is self limiting, two complications have been reported in literature. Lee et al, reported calcaneal avulsion fracture in four patients with neglected Sever's disease, ${ }^{10}$ and Mustapić et al. ${ }^{11}$ reported a case of Sever's disease complicated with calcaneal osteomyelitis.

Calcaneal osteomyelitis can result from either direct inoculation from a puncture wound, or from hematogenous spread from distant sites. ${ }^{12}$ In our patient, we could not exclude Sever's as a predisposing factor for such complication that may occur due to incomplete protection and continued repetitive trauma.

The ultrasonography is of limited value in diagnosis of osteomyelitis, However it is worthwhile for detecting complications such as sub-periosteal or soft tissue collections. ${ }^{12}$ On ultrasonography, 
subperiosteal collections are seen as periosteal elevation with a layer of echogenic fluid. While soft tissue abscesses appear as echogenic fluid collections, which may extend around the bony contours. Oedema and increased vascularity of soft tissue adjacent to bone may also be seen. ${ }^{13}$

In our patient the ultrasonography together with the X-ray provided strong indications to repeat the MRI. Furthermore the ultrasonography makes it useful for guiding needle aspiration of the collected fluid that confirmed its purulent nature.

\section{Conclusion}

Sever's disease is a common disorder among physically active children and adolescent. It is usually responding well to conservative treatment. However, it rarely complicates with calcaneal osteomyelitis. The clinicians should be aware of such rare complication that may result in great disability. The ultrasonography has a valuable role in assessment of soft tissues that may attribute to the heal pain. In our patient the ultrasonography was able to detect indirect signs of osteomyelitis in addition it guided the pus aspiration. These findings provided a strong base to repeat the MRI.

\section{Acknowledgments}

None.

\section{Conflict of interest}

Author declares no conflict of interests.

\section{References}

1. Hendrix CL. Calcaneal apophysitis (Sever disease). Clin Podiatr Med Surg. 2005;22(1): 55-62.
2. Sever JW. Apophysitis of the oscalcis. New York Med. 1912;95:1025-1029.

3. Almaawi A, Boszczyk AM, Daniels TR. Exostosis (Osteochondrosis, Apophysites, and Haglund's Deformity). In: Valderrabano V, Easley M, editors. Foot and Ankle Sports Orthopaedics. Springer International Publishing Switzerland. 2016; p. 209-216.

4. Hoşgören B, Köktener A, Dilmen G. Ultrasonography of the calcaneus in Sever's disease. Indian Pediatr. 2005;42(8):801-3.

5. Kose O. Do we really need radiographic assessment for the diagnosis of non-specific heel pain (calcaneal apophysitis) in children? Skeletal Radiol. 2010;39(4):359-361.

6. Lawrance DA, Rolen MF, Morshed KA, et al. MRI of Heel Pain. AJR. 2013;200:845-855.

7. Mc Millan AM, Landorf KB, Barrett JT, et al. Diagnostic imaging for chronic plantar heel pain: a systematic review and meta-analysis. $J$ Foot Ankle Res. 2009;2(1):1-11.

8. Bianchi S, Martinoli C, Abdelwahab IF. Ultrasound of tendon tears. Part 1: General considerations and upper extremity. Skeletal Radiol. 2005;34(9):500-512.

9. Cho KH, Lee YH, Lee SM, et al. Sonography of bone and bone-related diseases of the extremities. J Clin Ultrasound. 2004;32(9):511-521.

10. Lee KT, Young KW, Park YU, et al. Neglected Sever's Disease as a Cause of Calcaneal Apophyseal Avulsion Fracture: Case Report. Foot Ankle Int. 2010;31(8):725-728.

11. Mustapić M, Borić I, Lepur D, et al. Sever's disease complicated with osteomyelitis. Acta Clin Croat. 2014;53(2):252-255.

12. Malcius D, Jonkus M, Kuprionis G, et al. The accuracy of different imaging techniques in diagnosis of acute hematogenous osteomyelitis. Medicina (Kaunas). 2009;45(8):624-631.

13. Lee YJ, Sadigh S, Mankad K, et al. The imaging of osteomyelitis. Quant Imaging Med Surg. 2016;6(2):184-198. 